\title{
ROBUSTNESS ANALYSIS ON LATTICE-BASED SPEECH INDEXING APPROACHES WITH RESPECT TO VARYING RECOGNITION ACCURACIES BY REFINED SIMULATIONS
}

\author{
Yi-cheng Pan, Hung-lin Chang and Lin-shan Lee \\ Graduate Institute of Computer Science and Information Engineering, National Taiwan University \\ thomas02@iis.sinica.ed.tw, komkon@speech.ee.ntu.edu.tw, lslee@gate.sinica.edu.tw
}

\begin{abstract}
We analyze the robustness of different lattice-based speech indexing approaches. While we believe such analysis is important, to our knowledge it has been neglected in prior works. In order to make up for the lack of corpora with various noise characteristics, we use refined approaches to simulate feature vector sequences directly from HMMs, including those with a wide range of recognition accuracies, as opposed to simply adding noise and channel distortion to the existing noisy corpora. We compare, analyze, and discuss the robustness of several state-of-the-art speech indexing approaches.

Index Terms: spoken document retrieval, simulation
\end{abstract}

\section{INTRODUCTION}

Spoken document retrieval — or speech indexing — has been an active research topic in recent years; the recent NIST Spoken Term Detection (STD) evaluation is one example [1]. Many approaches have been proposed that take into account possible recognition errors by utilizing multiple speech recognition output alternatives in addition to the 1-best results. Lattice-based approaches such as position specific posterior lattices (PSPL) [2] and confusion networks (CN) [3, 4, 5] are good examples. These approaches have been analyzed in terms of accuracy and/or recall, space efficiency, and system response time. However, even if it is generally accepted that the performance of such approaches depends heavily on recognition accuracy, to our knowledge this has been somewhat neglected in the literature. We believe this issue is indeed important, since we wish to gauge performance given various recognition accuracies, as well as the robustness of various lattice-based speech indexing approaches.

In the Aurora 2.0 test environment for robust speech recognition, it is common to add noise and channel distortions to clean speech data to generate corpora with different disturbances. For speech indexing approaches, however, this approach proves inadequate, since such tasks usually are connected with real-world data such as lectures or broadcast conversation, for which clean speech data does not exist. Thus, as we may create corpora with more noise, but not with less, it is impossible to the assess the exact relationship between higher recognition accuracy and performance.

We describe a refined simulation procedure for producing lattices with varying recognition accuracies. Since hidden markov models (HMMs) are generative models, it is possible to generate feature vectors directly, by randomly choosing the HMM state and corresponding Gaussian mixture, and using these to generate samples for a given Gaussian distribution. To simulate corrupted data for varying recognition accuracies, we borrow a concept from uncertainty decoding [6] in which each observed feature vector is considered a random variable with a given distribution: by adjusting the variance of this distribution we control the quality of the resultant simulated feature vectors. Then, by performing recognition using these feature vectors, we can easily generate lattice corpora with a wide range of recognition accuracies. Thus we reduce the complicated problem of considering different types and levels of disturbances, as in robust speech recognition, to a single parameter: speech recognition accuracy. We then analyze the performance of each speech indexing approach with respect to this parameter. Because speech indexing is a higher-level process than recognition, this is reasonable.

The recently proposed techniques of subword-based PSPL (SPSPL) and subword-based CN (S-CN) [7] effectively reduce index size and at the same time significantly improve retrieval performance for both in-vocabulary (IV) and out-of-vocabulary (OOV) queries. In this paper we compare the robustness of these approaches with that of the conventional word-based PSPL (W-PSPL) and CN (W-CN) approaches.

\section{REFINED SIMULATION TECHNIQUES TO GENERATE CORPORA WITH DIFFERENT RECOGNITION ACCURACIES}

In this section, we present the refined simulation techniques to generate corpora with a wide range of recognition accuracies. For the sake of simplicity and feasibility, we only apply these techniques on HMMs with diagonal covariance matrices.

\subsection{Generation of Clean Feature Vectors from HMMs}

To generate a sequence of feature vectors conforming to the statistics depicted by an HMM, the first step is to generate a state sequence. This can be done by tossing a biased coin, each side of which determines whether to stay in the current state or not, with the biased probability set according to the transition probability. For each generated state in the state sequence, we then select a Gaussian distribution by tossing another biased multi-variate dice which has probabilities for each side according to the Gaussian mixture weights defined by the HMM. Finally we generate a sample feature vector from the Gaussian. Since we only consider Gaussian distributions with diagonal covariance matrices, we can generate each dimension of the sample feature vector independently. This can be easily achieved by Box-Muller transform [8]:

$$
z=\sigma \cdot \cos (2 \pi v) \sqrt{-2 \ln w}+\mu
$$

where $v$ and $w$ are two independent uniform random variables over $[0,1]$, and $z$ is the sample value generated from a Gaussian distribution with mean $\mu$ and variance $\sigma^{2}, \mathcal{N}\left(\mu, \sigma^{2}\right)$.

By concatenating HMMs together, we may generate clean feature vectors for a word, an utterance, a sentence, a paragraph and so on. The feature vectors generated in this way can be regarded as 
clean speech feature vectors since they are fully conforming to the underlying HMMs. When the feature vectors generated in this way based on the reference transcriptions of our Chinese broadcast news corpus using HMMs trained also with Chinese broadcast news with similar conditions, our recognizer gave a character accuracy of $94.0 \%$. This number is consistent with the result of Chinese broadcast news transcriptions obtained in GALE project [9].

\subsection{Generation of Corrupted Feature Vectors from HMM}

The approach here is borrowed from the techniques of uncertainty decoding [6]. We denote by $x$ the clean feature random variable, which can be modelled by a Gaussian $\mathcal{N}(\mu, \Sigma)$ in the given HMM, and by $\hat{x}$ the corrupted observed version of feature $x$. The difference between $x$ and $\hat{x}$ can be regarded as a random variable $e$, where $x=\hat{x}+e$. We assume that $e$ is a zero-mean Gaussian random variable,

$$
e \sim \mathcal{N}\left(0, \Sigma_{e}\right)
$$

It has been shown [6] that the probability distribution of $\hat{x}$ can be approximated by the Gaussian $\mathcal{N}\left(\mu, \Sigma+\Sigma_{e}\right)$, since the acoustic likelihood of observing $\hat{x}$, while considering all possible clean feature $x$, can be estimated by

$$
\int_{-\infty}^{\infty} \mathcal{N}(x ; \mu, \Sigma) \mathcal{N}\left(x ; \hat{x}, \Sigma_{e}\right) \mathrm{d} x
$$

where the second distribution $\mathcal{N}\left(x ; \hat{x}, \Sigma_{e}\right)$ in the integration is the probability distribution for $x$ given the observation $\hat{x}$, and the first distribution is the likelihood of $x$. It has been shown [6] the above integration over all possible values of $x$ can be reduced to

$$
\mathcal{N}\left(\hat{x} ; \mu, \Sigma+\Sigma_{e}\right)
$$

which is why the distribution of $\hat{x}$ can be approximated by the Gaus$\operatorname{sian} \mathcal{N}\left(\mu, \Sigma+\Sigma_{e}\right)$, or another Gaussian with the same mean, but the variance is increased by $\Sigma_{e}$.

With the above, the steps to generate corrupted sample vectors are exactly the same as in Sec. 2.1, except when generating the sample values for a given Gaussian, we add $\Sigma_{e}$ to the variance to generate the samples. By setting $\Sigma_{e}$ properly at different values, we can thus generate feature vectors with a wide range of recognition accuracies.

\section{LATTICE-BASED SPEECH INDEXING APPROACHES TO BE ANALYZED}

\subsection{Word-based Approaches}

In this section, we briefly summarize the original word-based position specific posterior lattices (PSPL) and confusion networks (CN) [2, 5, $4,3]$, both of which group the word arcs in the lattice into a sequence of strictly linear clusters, but in different ways. Each cluster includes several word arcs along with the corresponding posterior probabilities. Both approaches may produce proper soft-hit indices for each word in the spoken segments as represented by (word id, segment id, cluster number, posterior probability).

\subsubsection{Position-Specific Posterior Lattices (PSPL)}

The basic idea of PSPL is to calculate $P(W, k \mid L)$ as the posterior probability of a word $W$ at a specific position $k$ in a lattice $L$. Such information is actually hidden in the lattice $L$ because in each path of $L$ we clearly know each word's position. Since it is very likely that more than one path includes the same word at the same position, we need to aggregate the probabilities for a given word at a given position over all possible paths in a lattice. A variation of the standard forward-backward dynamic programming algorithm can be employed for this aggregation process [2].

\subsubsection{Confusion Networks (CN)}

Another approach was proposed earlier to cluster the word arcs in a word lattice using a completely different algorithm into several strictly linear and simple clusters of word alternatives, or the confusion networks $(\mathrm{CN})$ [3]. In each cluster, posterior probabilities for the word alternatives are also obtained. Although the original goal of $\mathrm{CN}$ was focused on the WER minimization for ASR, in speech indexing tasks we consider $\mathrm{CN}$ as a compact structure representing the original lattice, and it can also give us the proximity information of word arcs $[5,10,11,4]$.

\subsection{Subword-based Approaches}

Subword-based indexing approaches have been shown to be very helpful in speech indexing, since they can handle OOV and rare words to a good degree[12]. In this section we briefly summarize the previously proposed approaches, the subword-based PSPL (S-PSPL) [13] and $\mathrm{CN}$ (S-CN) [7] to incorporate subword information in both PSPL and CN structures.

\subsubsection{Subword-based Position Specific Posterior Lattices (S-PSPL)}

Similar to PSPL, the basic idea of S-PSPL is to calculate $P(w, k \mid L)$, which is the posterior probability of a subword $w$ at a specific position $k$ in a lattice $L$. Following the PSPL framework, it is easy to conduct a forward-backward algorithm to calculate $P(W, b, b+S u b(W)-1 \mid L)$ as the probability of a word $W$ being the $b^{\text {th }}$ to the $(b+S u b(W)-$ $1)^{\text {th }}$ subword units in the lattice $L$, where $S u b(W)$ is the number of subword units in $W$ [13]. Then by the assumptions made about subword posterior probabilities [13], we may have

$$
P(w, k \mid L)=\sum_{W, b} P(W, b, b+S u b(W)-1 \mid L),
$$

where $w$ is the $r^{\text {th }}$ subword in $W$ and $b+r-1=k$. In other words, the probability of a subword $w$ being the $k^{\text {th }}$ subword unit in the lattice is then simply the sum of the position specific posterior probabilities of those appropriate words $W$.

\subsubsection{Subword-based Confusion Network (S-CN)}

It is straightforward to construct a subword-based $\mathrm{CN}$ (S-CN) given the same approximations about posterior probabilities for subword units [13]. During ASR, we may record the start and end time for the subword units in each word arc. We then calculate the posterior probabilities for subword units using the assumptions in [13]. Regarding these subword units as subword arcs, we run the clustering algorithm just as the original word-based $\mathrm{CN}$ to construct $\mathrm{S}-\mathrm{CN}$. In each cluster of S-CN, we also sum up the posterior probabilities of subword arcs representing the same subword unit, as in the original $\mathrm{CN}$.

\section{EXPERIMENTS}

\subsection{Experimental Setup}

The corpus used in the experiments are the Mandarin broadcast news stories collected daily from local radio stations in Taiwan from August 
to September 2001. We manually segmented these stories into 5034 segments, each taken as a document. These 5034 segments are the same as those used in the previous works $[13,7]$, but here we primarily used the text parts (the manual transcriptions) to generate automatically the respective 5034 feature vector sequences. Since these segments are all short with very few silence frames, silence and short pauses were not considered here for simplicity. The 151 intrasyllable right-context-dependent initial-final acoustic models used to generate the feature vectors were trained with $8 \mathrm{hrs}$ of broadcast news stories collected in 2000, with cepstrum mean normalization applied. They were also used for the recognizer. Given the acoustic features, the decoding and indexing procedures are the same as the previous works $[13,7]$, using the same language model and lexicon. By setting different $\Sigma_{e}$ as described in Sec. 2.2, we simulated different sets of the feature vector sequences and therefore lattices and so on with different recognition accuracies. The character recognition accuracies obtained for these 5034 segments were controlled between $63 \%$ and 94\%. Since the corpus is in Mandarin Chinese, the subword units used in S-PSPL and S-CN can be either characters, syllables, or initial-final models. Here we only report the results on characters and syllables.

158 text test queries were generated by manual selection from a set of automatically generated candidates, each including 1 to 3 words. The candidates were high-frequency $n$-grams with length $1-3$ words which appeared at least 6 times in the 5034 segments. 38 of the 158 queries including OOV words were categorized as OOV queries (1.36 words and 3.60 characters long in average), while the remaining 120 were in-vocabulary (IV) queries ( 1.34 words and 3.04 characters long in average).

Because each Chinese word is composed of one to several characters, and each single character carries explicit meaning and can therefore also be considered as a mono-character word, a wellknown approach for handling OOV words in Chinese is to simply include all characters as mono-character words in the lexicon. Thus, when a query includes an OOV, we simply decompose it as a concatenation of in-vocabulary mono-character words or other words using the maximum matching algorithm in the case of word-based indexing and retrieval. For instance, for the threecharacter OOV $\left\{w_{1} w_{2} w_{3}\right\}$, the following decompositions are possible: $\left\{w_{1}\right\}\left\{w_{2}\right\}\left\{w_{3}\right\},\left\{w_{1}\right\}\left\{w_{2} w_{3}\right\}$, and $\left\{w_{1} w_{2}\right\}\left\{w_{3}\right\}$. In other words, for Chinese, OOV words are no longer OOV. After such preprocessing, the OOV queries are now 3.0 words long in average.

\subsection{Experimental Results}

All retrieval results presented here are in terms of Mean Average Precision (MAP), evaluated by the standard trec_eval package used by the TREC evaluations [14]. The MAP results for cases of OOV, IV and all queries are respectively shown Fig. 1, Fig. 2 and Fig. 3, each with six experiments: (a) W_P SPL: word-based PSPL, (b) C_P SP L: character-based PSPL, (c) S_P SP L: syllable-based PSPL, (d) W_CN: word-based CN, (e) C_CN: character-based CN, (f) S_CN: syllable-based $\mathrm{CN}$. We also included the six points representing the performances for the six cases of index structures (a)-(f) using the real 5034 speech segments, which had the true character recognition accuracy of $86.12 \%$.

In Fig. 1 of the OOV case, first we have the best performance from syllable-based approaches: (c) S_P SPL and (f) S_CN. This is expected since it is much easier to recognize OOV words as correct syllables than correct characters. Also, for either PSPL ((a)-(c)) or CN ((d)-(f)) respectively, syllable-based outperformed characterbased and character-based outperformed word-based $((c)>(b)>(a)$,

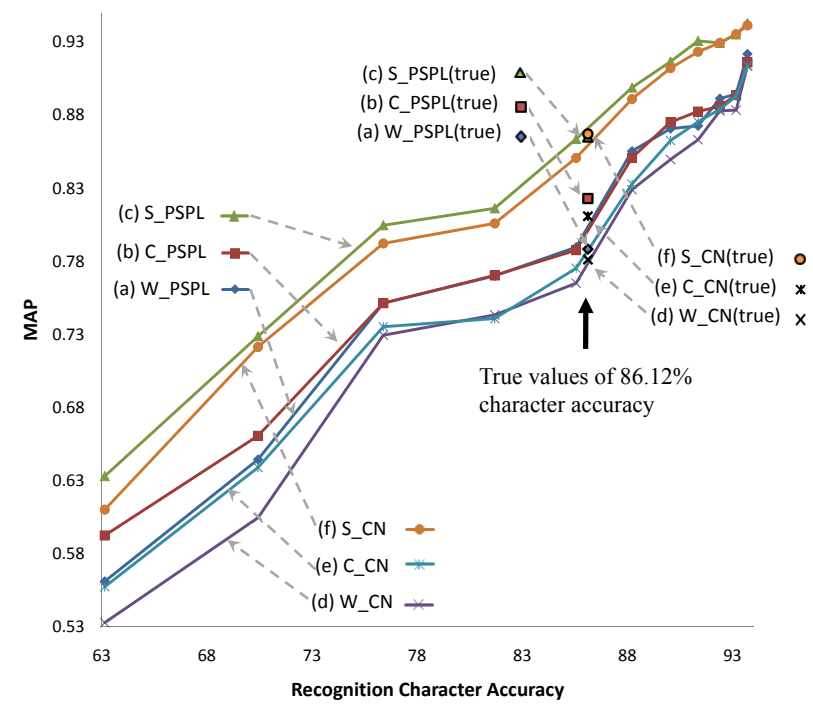

Fig. 1. MAP results for OOV queries.

$(\mathrm{f})>(\mathrm{e})>(\mathrm{d})$ ) in general. In addition, in most cases when based on the same units (syllable, character or words) PSPL was slightly higher than $\mathrm{CN}((\mathrm{a})>(\mathrm{d}),(\mathrm{b})>(\mathrm{e}),(\mathrm{c})>(\mathrm{f}))$. All these trends are consistent with those found previously with real speech data [7], and here we see these trends were actually consistent for a relatively wide range of recognition accuracy. Moreover, by comparing PSPL and CN in Fig. 1 ((a)-(c) vs. (d)-(f)) we see the differences between them were actually negligible at higher recognition accuracies, but the differences became more significant as the accuracy got worse. This is an interesting discovery, since this can be found only when we explore a wider range of accuracies by simulation. This is probably because when the accuracy is higher, we have much fewer word arcs with competitive probabilities in the word lattice, which usually have similar boundaries due to the good accuracy. This in turn alleviates the problems of arc clustering and missing paths for $\mathrm{CN}$ as discussed previously with real speech data [7]. So most of the paths can be found in the $\mathrm{CN}$ structures. This also implies $\mathrm{W}-\mathrm{CN} / \mathrm{S}-\mathrm{CN}$ becomes more attractive at higher recognition accuracies since $\mathrm{CN}$ generally requires less index size than PSPL, as also analyzed previously [7]. Also, we note that (a) W_P SPL and (b) C_P SPL (similarly for (d) W_CN and (e) C_CN) seem to have negligible differences, specially when the accuracies got higher. This is a departure from the original thoughts that subword-based approaches should offer improvements over wordbased approaches primarily for OOV cases. Note that the experiments here were on Chinese with the special OOV handling technique as described in Sec. 4.1; there are thus very little distinctions between word- and character-based approaches since very likely the OOV words are decomposed into several single characters in both the query and the recognized lattices. So the indexing structures of word- and character-based approaches around the OOV words are likely to be very similar. This is a special situation for Chinese with a good approach to handle OOV words. But C_P SPL/C_CN still has its role for Chinese as shown in Fig. 1 for lower accuracies, and also in the following IV case. Also shown in Fig. 1 are the values obtained by experiments with true speech data at character accuracy of $86.12 \%$. We see these points either almost exactly coincided with, or were very close to, the corresponding curves obtained by simulation, with exactly parallel trends. This verifies that the simulation approach here offered very good estimates for real situations. 


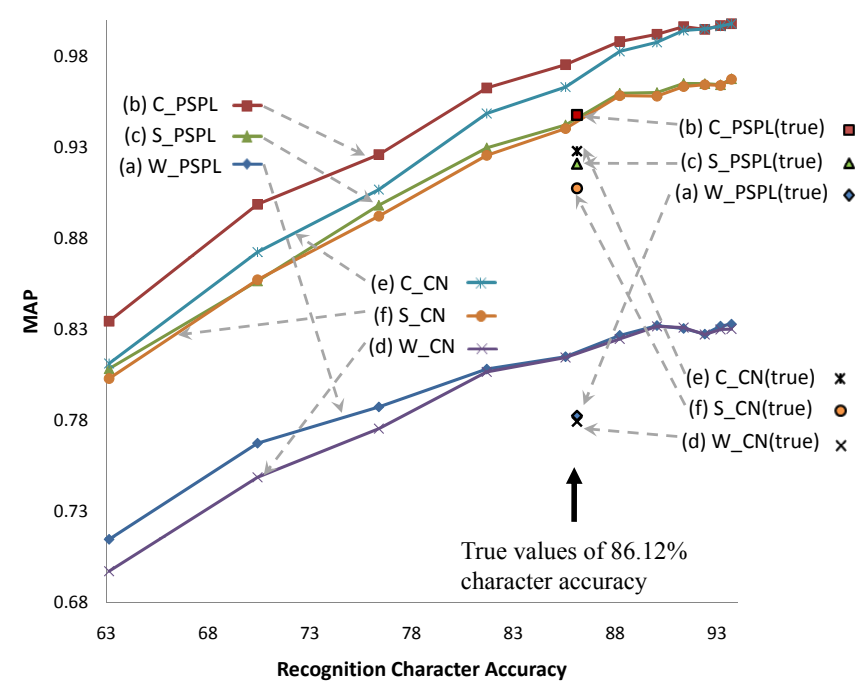

Fig. 2. MAP results for IV queries.

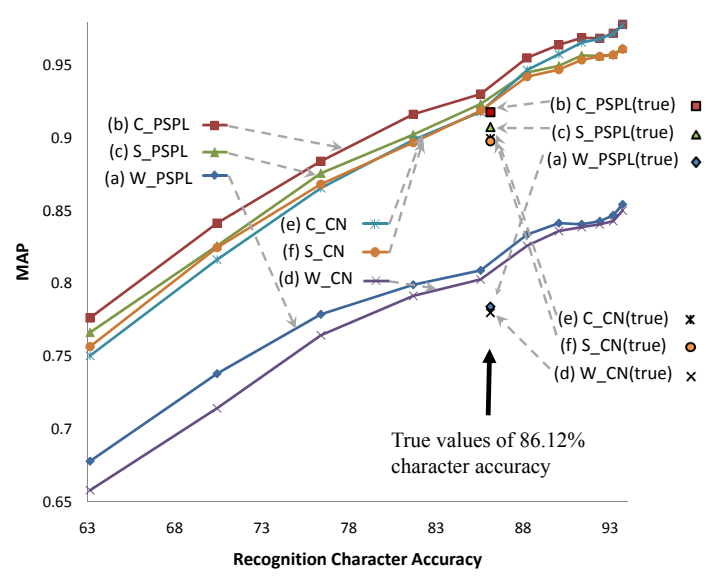

Fig. 3. MAP results for OOV+IV queries.

In Fig. 2 of IV case, the best performance was obtained from character-based approaches: (b) C_P SP L and (e) C_CN, since for IV the characters offer the most precise information. All other trends were very similar to those in Fig. 1 . All these are exactly consistent with earlier results and discussions based on real speech data [7]. An important new observation is that the slopes of subword-based approach curves are in general steeper than the word-based curves in most range of accuracies. This implies that subword-based approaches take better advantages when the accuracy improves. The word-based approaches improve only when some critical words are completely correctly recognized with high enough scores, while the subword-based approaches improve as long as some critical words are partially correctly recognized with high enough scores, which is more easier. So, if the recognition accuracy can be improved, significantly better retrieval performance can be expected for subword-based approaches. This may be another major advantage of subword-based approaches over word-based approaches which makes the subwordbased approach even more attractive, but cannot be found without using the simulation approach here. Also shown in Fig. 2 are the values obtained by experiments with true speech data at character accuracy of $86.12 \%$. Again the points showed exactly the same trends in parallel with those curves by simulation. However, all points here are slightly lower than the corresponding curves by almost the same gap. This may be explained as follows. In experiments with simulation almost all spoken segments have very close recognition accuracy in all cases. But for the real speech data, we found the accuracies for each spoken segment varied significantly, but with an average accuracy of $86.12 \%$. For those spoken segments with accuracies significantly lower than $86.12 \%$ the retrieval results can be much worse (the slope of the curve is even steeper there), so the average retrieval results were worse. This did not happen in the case of OOV queries in Fig. 1, probably because OOV queries were longer (average 3.0 words/3.60 characters for OOV queries but 1.34 words/3.0 characters for IV queries), so more information can be included, considered, and averaged.

Fig. 3 of the OOV+IV case shows the combined situation from Fig. 1 and Fig. 2 with basically the same trends. The overall best performance was from (b) C_P SPL which is also consistent with the previous results with real speech data [7].

\section{CONCLUSION}

In this paper, we make a first attempt to investigate the robustness issue for lattice-based speech indexing approaches, which is believed to be as important, but less touched in previous works. Instead of adding noise and channel distortions to existing corpus, we simulate feature vector sequences directly from HMMs, including generating a wider range of recognition accuracies. Several state-of-the-art latticebased speech indexing approaches are compared and some interesting observations are derived.

\section{REFERENCES}

[1] http://www.nist.gov/speech/tests/std/.

[2] C. Chelba, J. Silva, and A. Acero, "Soft indexing of speech content for search in spoken documents," Computer Speech and Language, vol. 21, no. 3, pp. 458-478, July 2007.

[3] L. Mangu, E. Brill, and A. Stolcke, "Finding consensus in speech recognition: Word error minimization and other applications of confusion networks," Computer Speech and Language, vol. 14, no. 2, pp. 373-400, 2000.

[4] T. Hori, I.L. Hetherington, T.J. Hazen, and J.R. Glass, "Open-vocabulary spoken utterance retrieval using confusion networks," in ICASSP, 2007, pp. 73-76.

[5] J. Mamou, D.Carmel, and R. Hoory, "Spoken document retrieval from call-center conversations," in SIGIR, 2006, pp. 51-58.

[6] Li Deng, Jasha Droppo, and Alex Acero, "Dynamic compensation of hmm variances using the feature enhancement uncertainty computed from a parametric model of speech distortion," SAP, vol. 13, no. 3, pp. 412-421, 2005.

[7] Y.-C. Pan, H.-L. Chang, and L.-S. Lee, "Analytical comparison between position specific posterior lattices and confusion networks based on words and subword units for spoken document indexing," in $A S R U, 2007$.

[8] G. E. P. Box and Mervin E. Muller, "A note on the generation of random normal deviates," The Annals of Mathematical Statistics, vol. 29, no. 2, pp. 610-611.

[9] M. Y. Hwang, G. Peng, W. Wang, A. Faria, A. Heidel, and M. Ostendorf, "Building a highly accurate mandarin speech recognizer," in ASRU, 2007, pp. 490-495.

[10] J. Mamou, B. Ramabhadran, and O. Siohan, "Vocabulary independent spoken term detection," in SIGIR, 2007, pp. 615-622.

[11] Ville T. Turunen and Mikko Kurimo, "Indexing confusion networks for morphbased spoken document retrieval," in SIGIR, 2007, pp. 631-637.

[12] K. Ng, Subword-based Approaches for Spoken Document Retrieval, Ph.D. thesis, Massachusetts Institute of Technology, 2000.

[13] Y.-C. Pan, H.-L. Chang, and L.-S. Lee, "Subword-based position specific posterior lattices (S-PSPL) for indexing speech information," in Interspeech, 2007.

[14] http://trec.nist.gov/. 